\title{
Respiratory chain enzyme defects in patients with idiopathic inflammatory myopathy
}

\author{
Yolanda Campos, Joaquin Arenas, Ana Cabello, Juan J Gomez-Reino
}

\begin{abstract}
Objective-To analyse muscle respiratory chain enzymes in idiopathic inflammatory myopathy.

Methods-Four consecutive female patients seen at our hospital with idiopathic inflammatory myopathy were studied. Muscle histochemical staining included NADH tetrazolium reductase and succinate dehydrogenase tests. Activity of rotenone sensitive NADH cytochrome $c$ reductase (complex $I$ and III) succinate dehydrogenase (complex II), succinate cytochrome $c$ reductase (complex II and III), cytochrome $c$ oxidase (complex IV), and citrate synthase (a mitochondrial matrix enzyme), was measured spectrophotometrically in muscle homogenates. Free carnitine, and short and long chain acylcarnitine esters were determined in muscle homogenates by a radiochemical procedure.

Results-Three patients had mitochondrial proliferation in nonregenerating muscle fibres; these patients had defects of respiratory chain enzyme complexes. Carnitine concentrations, measured in two of the four patients, revealed carnitine deficiency in one.

Conclusion-Our results suggest that mitochondrial dysfunction may be present in patients with inflammatory myositis.
\end{abstract}

(Ann Rheum Dis 1995; 54: 491-493)

Idiopathic inflammatory myopathies (IIM) are a heterogeneous group of disorders characterised by chronic muscle inflammation of unknown cause. ${ }^{1}$ IIM patients have proximal muscle weakness, increased serum concentrations of creatine kinase, typical electromyographic abnormalities, and mononuclear cell infiltration with necrosis and regeneration of myocytes in muscle biopsy specimens. ${ }^{1}$

Muscle of patients with mitochondrial disease shows both histological signs of mitochondrial proliferation and respiratory chain enzyme defects. ${ }^{2}$ Patients with IIM may also show mitochondrial proliferation in muscle biopsy specimens, defined by the presence of ragged red fibres or subsarcolemmal accumulation of oxidative elements. ${ }^{3}$

To our knowledge, biochemical analysis of muscle respiratory chain enzymes in patients with IIM has not been carried out. We present four patients with IIM, signs of muscle mitochondrial proliferation, and defect of respiratory chain enzymes.

\section{Patients and methods}

We studied four consecutive female patients who were seen at our hospital and for whom muscle biopsies were available. All met the criteria of Bohan and Peter for diagnosis of polymyositis. ${ }^{4}$ All four had proximal muscle weakness, increased blood concentrations of creatine kinase (CK) and typical electromyographic changes. $\mathrm{CK}$ values at diagnosis ranged from $1300 \mathrm{IU} / 1$ to $3690 \mathrm{IU} / 1$ (normal range < $150 \mathrm{IU} / \mathrm{l}$ ). All muscle biopsies showed inflammatory infiltrates, necrosis, and regeneration of fibres. One patient also had primary biliary cirrhosis (table 1 ). None of our patients had short stature, diabetes mellitus, mental retardation, seizures, cranial or peripheral nerve abnormalities, deafness or ophthalmoplegia at diagnosis; one patient developed diabetes mellitus while receiving treatment with corticosteroids. Patient 3 responded poorly to corticosteroids and required treatment with immunosuppressor drugs. The other three patients had a variable response to corticosteroids, but their disease was considered reasonably well controlled with different doses of corticosteroids.

Muscle histochemical staining included $\mathrm{NADH}$ tetrazolium reductase and succinate dehydrogenase tests and was by methods previously described. ${ }^{5}$ Activity of rotenone sensitive NADH cytochrome $c$ reductase (complex I and III), succinate dehydrogenase (complex II), succinate cytochrome $c$ reductase (complex II and III), cytochrome $c$ oxidase (complex IV), and citrate synthase (a mitochondrial matrix enzyme), was measured spectrophotometrically in muscle homogenates. ${ }^{6}$ Free carnitine, and short and

Table 1 Characteristics of four female patients with IIM

\begin{tabular}{lccll}
\hline Patient & $\begin{array}{c}\text { Age } \\
(y r)\end{array}$ & $\begin{array}{l}\text { Delay in } \\
\text { biopsy }^{\star}(y r)\end{array}$ & $\begin{array}{l}\text { Systemic } \\
\text { disease }\end{array}$ & $\begin{array}{l}\text { Previous } \\
\text { drugs } \$\end{array}$ \\
\hline 1 & 56 & 2 & $\begin{array}{l}\text { Pulmonary } \\
\text { fibrosis }\end{array}$ & - \\
2 & 72 & 11 & $\begin{array}{l}\text { Cardiac } \\
\text { Pulmonary }\end{array}$ & Steroids \\
3 & 41 & 2 & $\begin{array}{l}\text { fibrosis } \\
\text { Biliary } \\
\text { cirrhosis }\end{array}$ & Steroids \\
4 & 16 & 5 & - & - \\
\hline
\end{tabular}

^From initial symptoms. §Before biopsy; one patient was receiving steroids at the time of biopsy and in the other, steroids were discontinued several months before biopsy. 


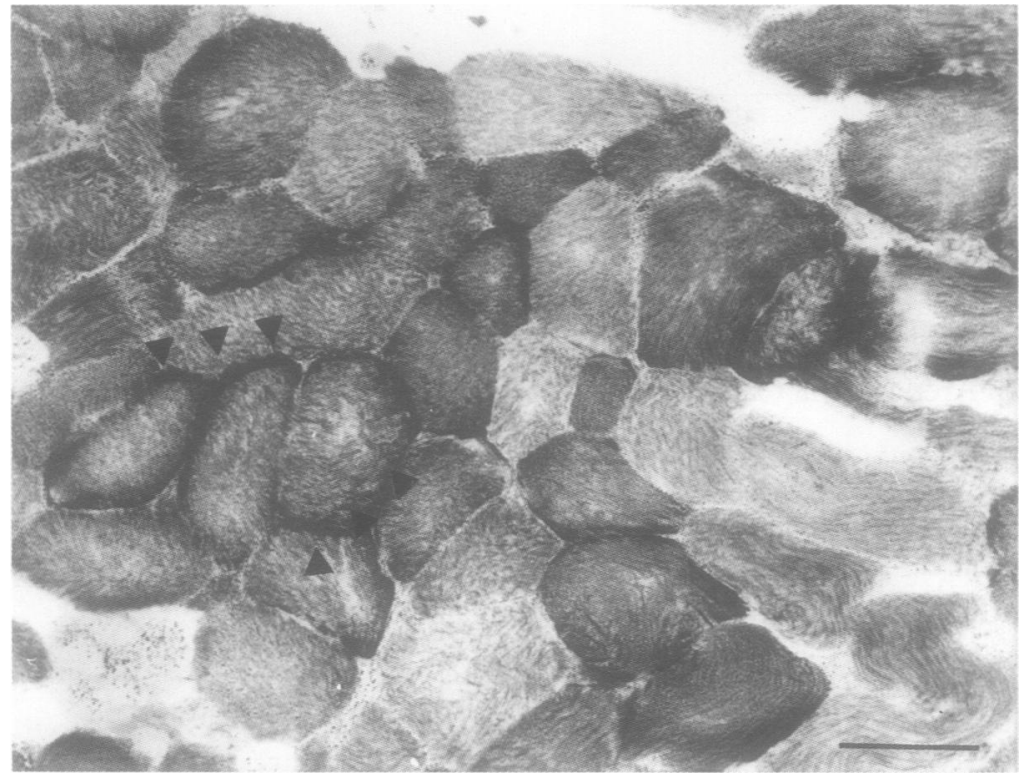

Typical muscle biopsy specimen showing increased subsarcolemmal oxidative accumulations of mitochondria (arrowheads) in non-regenerating type I fibres (staining with succinate dehydrogenase). Horizontal bar represents $50 \mu \mathrm{m}$.

long chain acylcarnitine esters were determined in muscle homogenates by a radiochemical procedure.

\section{Results}

The muscle biopsy specimens of three patients showed subsarcolemmal accumulations of oxidative elements in non-regenerating fibres with succinate dehydrogenase (figure) and trichrome staining. In one of these specimens, ragged red fibres were occasionally observed. Regenerating fibres showed mitochondrial proliferation in a diffuse non-subsarcolemmal pattern.

Table 2 shows the respiratory chain enzyme activities and carnitine concentrations. To correct for abnormal proliferation of mitochondria, the activity of each complex was normalised to the activity of citrate synthase, a good index of mitochondrial volume. Patient 1 , who showed neither ragged red fibres nor subsarcolemmal oxidative elements, had normal enzyme concentrations: patient 4 showed a defect of complex I, patient 3 of complex III, and patient 2 had multiple defects. Muscle concentrations of carnitine were determined in two patients: patient 1 had

Table 2 Respiratory chain enzyme activity and carnitine concentrations in muscle from four patients with idiopathic inflammatory myopathy and 25 healthy controls

\begin{tabular}{|c|c|c|c|c|c|}
\hline & \multirow{2}{*}{$\begin{array}{l}\text { Controls } \dagger \\
(n=25)\end{array}$} & \multicolumn{4}{|c|}{ Patients } \\
\hline & & 1 & 2 & 3 & 4 \\
\hline \multicolumn{6}{|l|}{ Complex } \\
\hline $\mathrm{I}+\mathrm{III}$ & $12(4)$ & $8 \cdot 5$ & 1.5 & $1 \cdot 8$ & $2 \cdot 5$ \\
\hline II + III & $7 \cdot 5(3)$ & 6 & $4 \cdot 2$ & $2 \cdot 1$ & $4 \cdot 7$ \\
\hline II & $10 \cdot 5(3 \cdot 5)$ & 4.5 & $1 \cdot 8$ & $6 \cdot 9$ & $6 \cdot 6$ \\
\hline IV & $53(17)$ & 50 & $4 \cdot 4$ & 20 & 97 \\
\hline \multicolumn{5}{|l|}{ Citrate synthase } & 135 \\
\hline \multicolumn{6}{|l|}{$\begin{array}{l}\text { Carnitine }(\mathrm{nmol} / \mathrm{mg} \\
\text { non-collagen protein) }\end{array}$} \\
\hline Free & $18.2(3.58)$ & $18 \cdot 5$ & nd & nd & $8 \cdot 6$ \\
\hline Short chain & $4.54(1.72)$ & $4 \cdot 2$ & nd & nd & $1 \cdot 8$ \\
\hline Long chain & $0.45(0.20)$ & $0 \cdot 4$ & nd & nd & 0.5 \\
\hline
\end{tabular}

The activity of each complex was normalised to that of citrate synthetase and expressed as percentage. + Values expressed as mean $(\mathrm{SD})$. nd $=$ Not done. normal carnitine values, while patient 4 showed carnitine deficiency.

\section{Discussion}

We have studied four patients with clinical, morphological, and electromyographic evidence of IIM. One had no evidence of mitochondrial proliferation in muscle and normal respiratory chain enzyme concentrations. Three had both histochemical evidence of mitochondrial proliferation and combined defects of the respiratory chain complexes, indicative of mitochondrial dysfunction. One of these patients also had primary biliary cirrhosis; this has been described previously in association with mitochondrial autoantibodies, ${ }^{8}$ which are directed to the pyruvate dehydrogenase complex rather than against respiratory chain components. ${ }^{8}$ Pyruvate dehydrogenase complex regulates the rate of pyruvate oxidation within mitochondria. Its deficiency is associated neither with mitochondrial proliferation nor with defects of the respiratory chain complexes. Yasuda et $a l^{4}$ described a patient with primary biliary cirrhosis whose clinical and morphological findings were similar to those of the patient we discuss here, but they did not report muscle respiratory chain enzyme concentrations.

Mitochondrial diseases are a heterogeneous group of disorders characterised histochemically by the presence of ragged red fibres or other signs of mitochondrial proliferation, and biochemically by single or combined defects of the respiratory chain complexes. ${ }^{2}$ Although morphological signs of mitochondrial dysfunction have been previously reported in muscle from patients with IIM, ${ }^{3}$ in biochemical analyses of respiratory chain complexes have not been documented. In our study, abnormal mitochondrial proliferation was evident in both regenerating and nonregenerating muscle fibres. It has been shown that, in damaged muscle, mononucleate satellite cells may proliferate and evolve into regenerating fibres that subsequently fuse to form myotubes. Thus mitochondrial proliferation in regenerating muscle cells from patients with IIM may be merely secondary to de novo formation of fibres, following necrosis. ${ }^{10}$ In non-regenerating fibres, mechanisms leading to mitochondrial proliferation remain unknown. It could be speculated that an imbalance between energy requirements and efficiency of oxidation/phosphorylation in muscle cells results in compensatory mitochondrial proliferation. In this regard, combined defects of the respiratory chain complexes were detected in non-regenerating muscle fibres from our three patients with mitochondrial proliferation.

Additional evidence for mitochondrial dysfunction comes from studies on muscle concentrations of carnitine. Carnitine has a key role in regulating the transport of long chain fatty acids into mitochondria and modulates intracellular concentrations of free coenzyme A. In plasma and tissues, carnitine is present in free form and as acylcarnitine esters. 
Patients with mitochondrial myopathy resulting from respiratory chain defects may progressively reduce their muscle carnitine pool and subsequently increase esterified carnitine. ${ }^{11}$ Interestingly, one of our patients with mitochondrial dysfunction had muscle carnitine deficiency, whereas another with normal mitochondrial function had normal carnitine concentrations. L-Carnitine therapy is claimed to be effective in patients with primary mitochondrial diseases, ${ }^{12}$ but the value of this therapy in patients with inflammatory myopathy and carnitine deficiency remains to be proved.

Finally, the response to steroids or immunosuppressors by patients with IIM is variable. A subset of patients is thought to be steroid resistent; they might represent a subgroup with an additional muscle defect. Our data show that respiratory chain enzyme defects can be present in a number of IIM patients; this deficit could lead to reduction in ATP concentrations which, if below a threshold level, may result in muscle weakness. Whether these patients are resistant to steroid therapy is unknown at present. Further work may help to clarify this issue.

This work was supported by an FIS grant (92/0363), Ministry of Health, Spain. Yolanda Campos was supported by SigmaTau, Spain.
1 Love L A, Leff R L, Fraser D D, et al. A new approach to the classification of idiopathic inflammatory myopathy: myositis-specific autoantibodies define useful homogeneous patients groups. Medicine 1991; 70: 360-74.

2 DiMauro S, Moraes C T. Mitochondrial encephalomyopathies. Arch Neurol 1993; 50: 1197-208. 3 Banker B Q, Engel A G. The polymyositis and dermatomyositis syndromes. In: Engel A G, Banker B Q,
eds. Myology. New York: McGraw Hill, 1986: $1385-422$.

4 Bohan A, Peter J B. Polymyositis and dermatomyositis. Part 1. N Engl f Med 1975; 292: 344-7.

5 Tristchler $\mathrm{H} \mathrm{J}$, Andreeta F, Moraes C T, et al. Mitochondrial myopathy of childhood associated with depletion of mitochondrial DNA. Neurology 1991; 42: 209-17.

6 DiMauro S, Servidei S, Zeviani M, et al. Cytochrome $c$ oxidase deficiency in Leigh syndrome. Ann Neurol 1987; 22: 498-506.

7 DiDonato S, Rimoldi M, Garavaglia B, Uziel G. Propionyl carnitine excretion in propionic and methylmalonic acidurias: a cause of carnitine deficiency. Clin Chim Acta 1984; 139: 13-2

8 Zurgil N, Bakimer R, Moutsopoulos H M, et al. Antimitochondrial (pyruvate dehydrogenase) autoantibodies in autoimmune rheumatic diseases. F Clin Immunol 1992; 12: 201-9.

9 Yasuda Y, Nakano S, Akiguchi I, Tanaka M, Kameyama M. Polymyositis associated with asymptomatic primary biliary cirrhosis. Eur Neurol 1993; 33: 51-3.

10 Watkins S C, Cullen M J. A qualitative and quantitative study of the ultrastructure of regenerating muscle fibers in Duchenne muscular dystrophy and polymyositis. in Neurol Sci 1987; 82: 181-92.

11 Campos Y, Huertas R, Bautista J, et al. Muscle carnitine deficiency and lipid storage myopathy in patients with mitochondrial myopathy. Muscle Nerve 1993; 16: 778-81.

12 Campos Y, Huertas R, Lorenzo G, et al. Plasma carnitine insufficiency and effectiveness of $\mathrm{L}$-carnitine therapy in patients with mitochondrial myopathy. Muscle Nerve 1993; 16: 150-3. 